$$
\cdot \mathfrak{h}^{1}=\sum_{i=1}^{n} \mathfrak{h}_{i}^{1}+(p-n-m+1) \mathfrak{g} .
$$

Proof. In constructing 1-simplexes $\left({ }^{\prime} 0^{\prime \prime} 0\right), \cdots,\left({ }^{\prime} 0^{(m)} 0\right)$ (not belonging to $\cdot \mathfrak{M}^{2}$, we get $\cdot \mathfrak{M}^{* 2}, \mathfrak{M}_{1}^{* 2}, \cdots$ as in the lemma. By $(7)$ and (8), we have

$$
\cdot \mathfrak{h}_{i}^{* 1}=\mathfrak{h}_{i}^{1}+\left(p_{i}-1\right) \mathfrak{g}
$$

where $\cdot \mathfrak{h}_{i}^{* 1}$ is the homology group of $\cdot \mathfrak{M}_{t}^{* 2}$. The newly constructed simplexes form a connected 1-complex whose 1-dimensional homology group contains the identity only. Therefore from a famous theorem (cf. Seifert-Threfall, p. 179), by (5) we get

$$
\cdot \mathfrak{h}^{* 1}=\sum_{i=1}^{n} \mathfrak{h}_{i}^{1}+(p-n) \mathfrak{g},
$$

where $\mathfrak{h}^{* 1}$ is the homology group of $\cdot \mathfrak{M}^{* 2}$. Therefore (9) is finally established in virtue of (11) and (8)'.

Theorem (3.5) may be extended analogously.

NATIONAL WUHAN UnIVERSity

\title{
A NOTE ON EQUICONTINUITY
}

M. K. FORT, JR.

During a recent seminar discussion of his paper Transitivity and equicontinuity [1], ${ }^{1}$ W. H. Gottschalk proposed the following question:

"Is the center of every algebraically transitive group of homeomorphisms on a compact metric space equicontinuous?"

An affirmative answer to the above question is given in this note.

1. Definitions. We let $X$ and $Y$ be compact metric spaces and let $d$ be the metric for $Y$.

A set $F$ of functions on $X$ into $X$ is algebraically transitive if corresponding to each pair $p$ and $q$ of points in $X$ there exists $f \in F$ such that $f(p)=q$.

A sequence $\left[g_{n}\right]$ of functions on $X$ into $Y$ converges to a function

Presented to the Society, November 27, 1948; received by the editors August 10, 1948.

${ }^{1}$ Numbers in brackets refer to the bibliography at the end of the paper. 
$g$ uniformly at a point $p \in X$ if $\epsilon>0$ implies that there exists $N>0$ and a neighborhood $V$ of $p$ such that $d\left(g_{n}(x), g(x)\right)<\epsilon$ whenever $x \in V$ and $n>N$.

We shall need to make use of the fact that if $\left[g_{n}\right]$ is a sequence of continuous functions on $X$ into $Y$ which converges pointwise to a function $g$ on $X$, then the sequence converges uniformly at each point of a set residual in $X$. This fact has been proved by Kuratowski in [2]. Although the notation implies that Kuratowski is restricting himself to more special spaces than those with which we are dealing, the proof given in [2] is actually valid for any compact metric spaces $X$ and $Y^{2}$

2. A more general theorem. We shall now prove a theorem which yields as a corollary the answer to Gottschalk's question.

THeOREM. Let $F$ be a set of continuous functions on $X$ into $X$ and $G$ a set of continuous functions on $X$ into $Y$, such that to each $f \in F$ there corresponds a continuous function $f^{*}$ on $Y$ into $Y$ such that $g=f^{*} g f$ for all $g \in G$. If $F$ is algebraically transitive then $G$ is equicontinuous.

Proof. It is well known that in order to prove $G$ equicontinuous it is sufficient to prove that every sequence in $G$ has a uniformly converging subsequence. This is the converse of Ascoli's theorem.

Let $S$ be any sequence in $G$. Choose a subsequence $\left[g_{n}\right]$ of $S$ which converges at some point $p \in X$. This is possible since $Y$ is compact. We shall prove that $\left[g_{n}\right]$ converges uniformly on $X$.

We first prove that $\left[g_{n}\right]$ converges pointwise on $X$. Let $x$ be any point of $X$. Since we are assuming that $F$ is algebraically transitive, we may choose $f \in F$ such that $f(x)=p$. There exists, by hypothesis, a continuous function $f^{*}$ on $Y$ into $Y$ such that $g=f^{*} g f$ for all $g \in G$. Since $f(x)=p$ and $\left[g_{n}\right]$ converges at $p$, we see that $\left[g_{n} f(x)\right]$ is a converging sequence in $Y$. Since $f^{*}$ is continuous on $Y$, it follows that the sequence $\left[f^{*} g_{n} f(x)\right]$ converges. This sequence is the same, however, as $\left[g_{n}(x)\right]$.

Since we now know that $\left[g_{n}\right]$ converges pointwise on $X$, we may let $g_{0}$ be the limit of the sequence of functions. The sequence $\left[g_{n}\right]$ converges to $g_{0}$ uniformly at each point of a set residual in $X$, and since $X$ is a compact metric space this residual set is non-empty. Let $q$ be a point at which $\left[g_{n}\right]$ converges uniformly to $g_{0}$.

${ }_{2}^{2}$ The theorem is true for functions on any topological space $X$ into a separable metric space $Y$. The author has a proof of this fact which will be included in a later paper on applications of semi-continuous set-valued functions. 
We now prove that $\left[g_{n}\right]$ converges uniformly at each point of $X$. Let $x$ be a point of $X$ and choose $f \in F$ such that $f(x)=q$. There exists $f^{*}$, continuous on $Y$ into $Y$, such that $g=f^{*} g f$ for all $g \in G$. The function $g_{0}$ may not belong to $G$, but since $g_{0}$ is the pointwise limit of a sequence of elements of $G$ it is easy to verify that $g_{0}=f^{*} g_{0} f$. Suppose $\epsilon>0$. There exists $\delta>0$ such that if $u$ and $v$ belong to $Y$ and $d(u, v)<\delta$ then $d\left(f^{*}(u), f^{*}(v)\right)<\epsilon$. There exists $N>0$ and a neighborhood $U$ of $q$ such that $d\left(g_{n}(y), g_{0}(y)\right)<\delta$ whenever $n>N$ and $y \in U$. There exists a neighborhood $V$ of $x$ such that $f(V) \subset U$. It is now easy to see that if $z \in V$ and $n>N$ then $d\left(f^{*} g_{n} f(z), f^{*} g_{0} f(z)\right)<\epsilon$. We thus obtain $d\left(g_{n}(z), g_{0}(z)\right)<\epsilon$ whenever $z \in V$ and $n>N$. This proves that the convergence is uniform at $x$.

If a sequence of functions converges uniformly at each point of a compact space, then the sequence converges uniformly on the entire space. Therefore $\left[g_{n}\right]$ converges uniformly to $g_{0}$ on $X$.

COROLlaRY 1. If $F$ is an algebraically transitive group of homeomorphisms of $X$ onto $X$ and $G$ is a group of homeomorphisms of $X$ onto $X$ such that $g f=f g$ whenever $f \in F$ and $g \in G$, then $G$ is equicontinuous.

COROLlaRY 2. If $F$ is an algebraically transitive group of homeomorphisms of $X$ onto $X$, then the center of $F$ is equicontinuous.

\section{BIBLIOGRAPHY}

1. W. H. Gottschalk, Transitivity and equicontinuity, Bull. Amer. Math. Soc. vol. 54 (1948) pp. 982-984.

2. C. Kuratowski, Sur les fonctions représentables analytiquement et les ensembles de première catégorie, Fund. Math. vol. 5 (1924) pp. 75-91.

UNIVERSITY OF VIRGINIA 